ARTICLE

https://doi.org/10.1038/s41467-018-07875-7

\title{
Oxygen-dependent proteolysis regulates the stability of angiosperm polycomb repressive complex 2 subunit VERNALIZATION 2
}

\author{
Daniel J. Gibbs (10 1, Hannah M. Tedds (10 1, Anne-Marie Labandera1, Mark Bailey', Mark D. White ${ }^{2}$,
} Sjon Hartman (10 ${ }^{3}$, Colleen Sprigg ${ }^{1}$, Sophie L. Mogg (1) ${ }^{1}$, Rory Osborne ${ }^{1}$, Charlene Dambire ${ }^{4}$, Tinne Boeckx ${ }^{4}$, Zachary Paling ${ }^{1}$, Laurentius A.C.J. Voesenek ${ }^{3}$, Emily Flashman ${ }^{2} \&$ Michael J. Holdsworth (1) 4

The polycomb repressive complex 2 (PRC2) regulates epigenetic gene repression in eukaryotes. Mechanisms controlling its developmental specificity and signal-responsiveness are poorly understood. Here, we identify an oxygen-sensitive $\mathrm{N}$-terminal $(\mathrm{N}-$ ) degron in the plant PRC2 subunit VERNALIZATION(VRN) 2, a homolog of animal Su(z)12, that promotes its degradation via the $\mathrm{N}$-end rule pathway. We provide evidence that this $\mathrm{N}$-degron arose early during angiosperm evolution via gene duplication and $\mathrm{N}$-terminal truncation, facilitating expansion of PRC2 function in flowering plants. We show that proteolysis via the $\mathrm{N}$-end rule pathway prevents ectopic VRN2 accumulation, and that hypoxia and long-term cold exposure lead to increased VRN2 abundance, which we propose may be due to inhibition of VRN2 turnover via its $\mathrm{N}$-degron. Furthermore, we identify an overlap in the transcriptional responses to hypoxia and prolonged cold, and show that VRN2 promotes tolerance to hypoxia. Our work reveals a mechanism for post-translational regulation of VRN2 stability that could potentially link environmental inputs to the epigenetic control of plant development.

\footnotetext{
${ }^{1}$ School of Biosciences, University of Birmingham, Edgbaston B15 2TT, UK. ${ }^{2}$ Chemistry Research Laboratory, University of Oxford, 12 Mansfield Road, Oxford OX1 3TA, UK. ${ }^{3}$ Plant Ecophysiology, Institute of Environmental Biology, Faculty of Science, Utrecht University, Padualaan 8, Utrecht $3584 \mathrm{CH}$, The Netherlands. ${ }^{4}$ School of Biosciences, University of Nottingham, Loughborough LE12 5RD, UK. These authors contributed equally: Hannah M. Tedds, AnneMarie Labandera. Correspondence and requests for materials should be addressed to D.J.G. (email: d.gibbs@bham.ac.uk)

or to M.J.H. (email: michael.holdsworth@nottingham.ac.uk)
} 
P olycomb group $(\mathrm{PcG})$ proteins are essential regulators of gene expression in eukaryotes, functioning as multiprotein complexes to establish epigenetically silenced states on their gene targets ${ }^{1-3}$. One of the best characterized of these complexes is the polycomb repressive complex 2 (PRC2), which catalyses the deposition of the repressive histone $\mathrm{H} 3$ lysine 27 trimethylation (H3K27me3) mark on chromatin ${ }^{4,5}$. This modification is mitotically stable and therefore acts as a long-term (yet reversible) suppressor of gene transcription. PRC2 activity controls cell identity, developmental transitions and the establishment of environmental memory across kingdoms ${ }^{4,5}$. Although many PRC2 functions and gene targets have been identified, mechanisms underpinning signal perception and transduction via this complex, as well as its developmental specificity, are still poorly understood.

The canonical PRC2 comprises four subunits: ENHANCER OF ZESTE [E(z)], SUPRESSOR OF ZESTE 12 [Su(z)12], EXTRA SEX COMBS (Esc) and p55 $2,4,5 . \mathrm{E}(\mathrm{z})$ is the catalytic component with histone-methyltransferase (HMTase) activity, whilst $\mathrm{Su}(\mathrm{z}) 12$ and Esc are both required for complex integrity and facilitating methylation by $\mathrm{E}(\mathrm{z})^{2,6}$. A wide range of context-specific binding partners also contribute to specificity, efficiency and robustness of PRC2 activity ${ }^{2,5}$. In contrast to animals, plants encode multiple copies of PRC2 subunits ${ }^{5,7}$, allowing the formation of different complexes with distinct functions, although knowledge of how and why these components diverged during evolution to acquire new roles is limited. In Arabidopsis thaliana, PRC2 complexes are classified depending on which one of three $\mathrm{Su}(\mathrm{z}) 12$-like subunits they recruit: FERTILIZATION INDEPENDENT 2 (FIS2), EMBRYONIC FLOWER 2 (EMF2) or VERNALIZATION 2 $(\mathrm{VRN} 2)^{5,7,8}$. FIS2-PRC2 inhibits seed development in the absence of fertilization ${ }^{9}$, whilst EMF2-PRC2 promotes vegetative growth through suppressing flowering ${ }^{10}$. VRN2-PRC2 has several developmental functions ${ }^{11-13}$, but is best known as a key regulator of vernalization, the epigenetic process by which long-term cold exposure promotes the transition from vegetative to reproductive development ${ }^{14}$. During vernalization, VRN2-PRC2 methylates and silences the floral repressor gene FLOWERING LOCUS C (FLC), thereby encoding a memory of cold that permits flowering once warm temperatures return. A molecular mechanism regulating the cold-triggered accumulation ${ }^{15}$ of VRN2 required for this process is not known.

The evolutionarily conserved $\mathrm{N}$-end rule pathway regulates protein destruction through the recognition of N-terminal degradation sequences ( $\mathrm{N}$-degrons) in target proteins, which promote their ubiquitylation by specific E3 ligases (N-recognins) ${ }^{16-18}$. Several branches of the $\mathrm{N}$-end rule pathway are known, which mediate a broad range of growth, developmental and stress-associated processes across kingdoms (reviewed in refs. ${ }^{16,17}$ ). In plants, group VII ETHYLENE RESPONSE FACTOR (ERFVII) transcription factors are Methionine-Cysteine- (Met-Cys-; MC-) initiating substrates of the arginylation (Arg)/N-end rule pathway ${ }^{19-21}$, which are regulated by oxygen $\left(\mathrm{O}_{2}\right)$ and nitric oxide (NO), similar to several REGULATOR OF G PROTEIN SIGNALLING (RGS) proteins in mammals ${ }^{22,23}$. ERFVIIs are targeted for destruction in normoxia via $\mathrm{O}_{2}$-dependent oxidation of Nt-Cys, which involves the successive actions of METHIONINE AMINOPEPTIDASES, PLANT CYSTEINE OXIDASES (PCOs) ${ }^{24,25}$, ARGINYL TRANSFERASES (ATEs), and recognition by the N-recognin PROTEOLYSIS6 (PRT6) (summarized in Fig. 1a). NO is also required for degradation $^{21}$, although whether it is involved in Nt-Cys oxidation, or acts indirectly via other factors is currently not known. Under low- $\mathrm{O}_{2}$ conditions (hypoxia), Nt-Cys oxidation is limited, leading to increased protein accumulation and function. Thus, ERFVII stability and activity is coupled to $\mathrm{O}_{2}$ (and NO) availability, which is important for regulating the survival of flooding stress, other abiotic and biotic stresses, and photomorphogenesis ${ }^{26-29}$. We hypothesized that hypoxia may regulate further aspects of development or environment-response through modulating the stability of other MC-initiating regulatory proteins with different cellular functions.

Here, we identify the plant PRC2 subunit VRN2 as a substrate of the Arg/N-end rule pathway via its conserved $\mathrm{N}$-terminal Cys2 residue. Despite constitutive VRN2 expression, this posttranslational regulation confines VRN2 to the meristems of roots and shoots under non-vernalizing and aerobic growth conditions. We show that both submergence-induced hypoxia and long-term cold exposure lead to enhanced VRN2 accumulation throughout the plant. Cold-exposure induced several transcriptional and post-translational changes typically associated with hypoxia, including ERFVII stabilization and accumulation of hypoxia-related transcripts, suggesting overlap in the cellular response to both conditions. Thus our work identifies the $\mathrm{N}$-end rule pathway as a key determinant of tissue-specific and environment-responsive VRN2-PRC2 activity. Furthermore, phylogenetic and biochemical analyses provide evidence that the $\mathrm{N}$-degron of VRN2 was exposed early in angiosperm evolution through gene duplication and $\mathrm{N}$-terminal truncation of an ancient homolog, which facilitated the expansion of PRC2 function in plants through linking the stability of $\mathrm{Su}(\mathrm{z}) 12$ to the perception of the environment.

\section{Results}

VRN2 is a substrate of the Arg/N-end rule pathway. We identified VRN2 as a candidate target $\mathrm{N}$-end rule substrate, due to its MC-initiating N-terminus, which is conserved throughout angiosperms (Fig. 1b). Wild type (WT) VRN2-HA expressed in an in vitro rabbit reticulocyte system (containing functional $\mathrm{N}$-end rule pathway components ${ }^{19}$ ) was degraded following treatment with the translation inhibitor cycloheximide $(\mathrm{CHX})$, whereas co-treatment with the proteasome inhibitor bortezomib (BZ), or mutation of Cys2 to Alanine (Ala; a stabilizing residue), prevented this turnover (Fig. 1c). VRN2-FLAG ${ }^{15}$ was also unstable in transgenic seedlings, but stabilized by BZ, indicating it is regulated via the $26 \mathrm{~S}$ proteasome in vivo (Fig. 1d). To test if this regulation is linked to the $\mathrm{N}$ end rule pathway, we introduced VRN2-FLAG into prt6-1 (that lacks PRT6 E3 ligase activity ${ }^{19}$ ), and observed increased protein accumulation compared to WT (Fig. 1d). Next, we generated a series of transgenic plants expressing WT or mutant (Ala2) VRN2GUS ( $\beta$-glucuronidase) fusions driven by approximately $2 \mathrm{~kb}$ of the native VRN2 promoter. Similarly to VRN2-FLAG, we observed accumulation of VRN2-GUS in both prt6-1 and ate1 ate2 (that lacks ATE activity ${ }^{19}$ ), as well as higher steady-state levels of mutant Ala2-VRN2-GUS protein relative to WT VRN2-GUS in Col-0 (Fig. 1e-g and Supplementary Figure 1a, b). Transgene-specific RTPCR showed that there were no significant differences in WTVRN2-GUS or Ala2-VRN2-GUS expression in the different genetic backgrounds, confirming that these changes in abundance relate to post-translational control, and published microarray data ${ }^{19}$ confirmed that endogenous VRN2 mRNA levels are also similar across genotypes (Fig. 1h). Histochemical staining of 7-day-old seedlings showed that VRN2-GUS in WT is largely confined to the meristematic regions of shoots and roots (Fig. 1i, j and Supplementary Figure $2 \mathrm{a}, \mathrm{b})$. In contrast, treatment with BZ, expression in prt6-1, or an Ala2 mutation, all led to enhanced stability and a much broader domain of accumulation throughout seedlings and adult plants (Fig. 1i, j and Supplementary Figure 2). This reveals that the $\mathrm{N}$-end rule pathway confines VRN2 accumulation to meristematic tissues, despite constitutive VRN2 RNA expression ${ }^{19}$ (Supplementary Figure 3). 


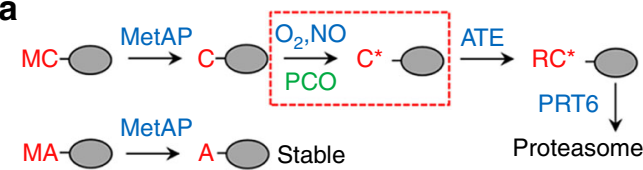

b

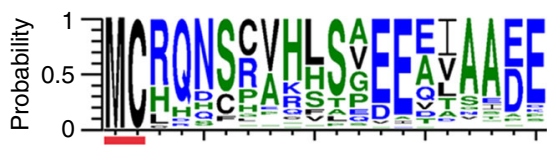

C

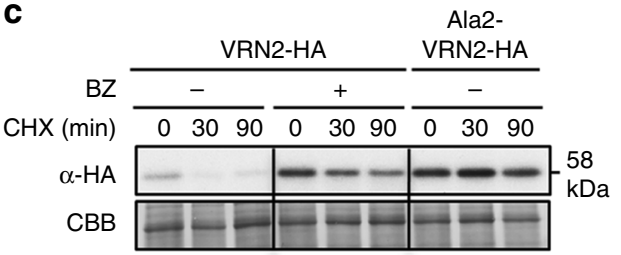

$\mathbf{e}$

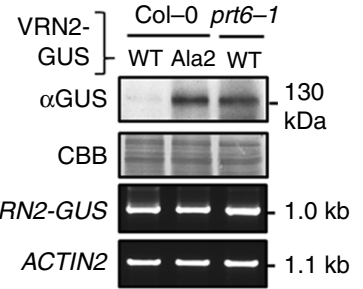

f

d
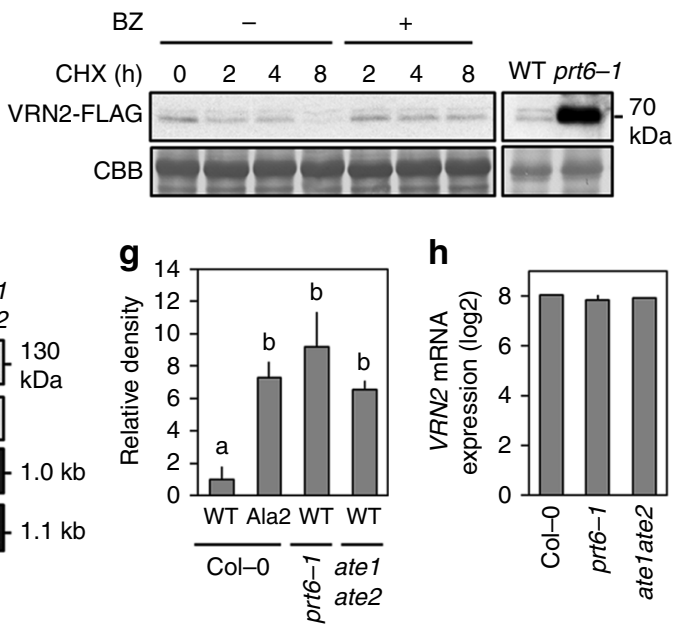

h

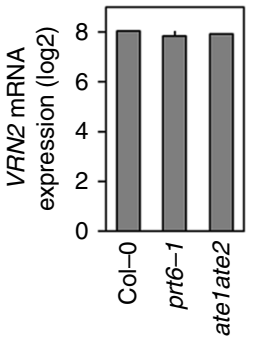

j VRN2-GUS

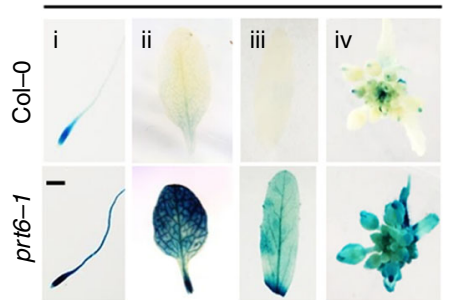

Fig. 1 VRN2 is a substrate of the Arg/N-end rule pathway of proteolysis. a N-terminal processing events leading to degradation of Met-Cys- (MC)-proteins via the $\operatorname{Arg}(\mathrm{R}) / \mathrm{N}$-end rule pathway: MetAP methionine aminopeptidase, PCO plant cysteine oxidase (specific to plants), ATE arginyl transferase, PRT6 proteolysis 6, C* Cys-sulfinic acid (oxidised Cys); red box is gas-dependent step. b Probability plot of AA by position in VRN2-like sequences derived from sequenced angiosperms following MUSCLE alignment, highlighting the conserved MC N-terminus (red bar). See Supplementary Data 2 for sequence data. c In vitro cycloheximide (CHX) chase of WT and mutant (Ala2) VRN2-HA ( \pm bortezomib; BZ). CBB Coomassie brilliant blue (showing equal loading). d CHX-chase of VRN2-FLAG in 7-day-old seedlings \pm BZ treatment, and steady state levels in prt6-1 vs WT. e, $\mathbf{f}$ Protein and mRNA levels of WT and mutant (Ala2) VRN2-GUS in Col-0, prt6-1 and ate1 ate2 seedlings. $\mathbf{g}$ VRN2-GUS protein accumulation in Col-0, prt6-1 and ate1 ate2 shown as relative density. Data is averaged from western blots for three independent lines for each transgene (see also Supplementary Figure 1a and b). Letters indicate one-way ANOVA; Tukey's test. h VRN2 mRNA expression levels in Col-0, prt6-1 and ate1 ate2, taken from published microarray data ${ }^{19}$. i Histochemical staining of 7-day-old Col-0 or prt6-1 seedlings expressing WT or mutant (Ala2) VRN2-GUS \pm BZ. Lines are the same as those presented in (e) and (f). Scale bar $500 \mu \mathrm{m}$. See also supplementary Figure 2a for biological reps. $\mathbf{j}$ Histochemical staining of (i) 7-day-old seedling primary root tip, (ii) rosette leaf, (iii) cauline leaf and (iv) inflorescence of Col-0 and prt6-1 lines expressing WT VRN2-GUS. Scale bar in (i), $500 \mu \mathrm{m}$. Source data are provided as a Source Data file

VRN2 is regulated by $\mathrm{O}_{2}$ and contributes to hypoxia tolerance. Since degradation of VRN2 via the $\mathrm{N}$-end rule pathway is dependent on its Cys2 residue, we examined the influence of $\mathrm{O}_{2}$ and NO on VRN2 stability. We observed strong posttranslational accumulation of VRN2-FLAG and VRN2-GUS in seedlings exposed to submergence-induced hypoxia or the NO scavenger cPTIO (Fig. 2a-d and Supplementary Figure 2a). We also found that recombinant Arabidopsis PCOs could oxidize in vitro the $\mathrm{Nt}$-Cys residue of a peptide representing the Met1excised N-terminus of VRN2. Mass shifts of $32 \mathrm{Da}$ were observed in the presence of all 5 PCOs (Fig. 2e and Supplementary Figure $4 \mathrm{a}$ ), signifying $\mathrm{O}_{2}$-dependent Cys-sulfinic acid generation, which is required for subsequent ATE activity and recognition by PRT $6^{25}$. Previous work showed that prt6-1 has enhanced hypoxia resistance, due to the constitutive accumulation of ERFVIIs ${ }^{19}$. To assess if ectopic VRN2 accumulation in prt6-1 also contributes to this resilience, we isolated a new null T-DNA allele in Col-0, vrn2-5 (Supplementary Figure 5), and generated combination mutants for assessing seedling root tip survival following hypoxic stress. Survival of root tips under hypoxia was much greater in prt6-1 than Col-0, but was significantly reduced in the prt6-1 vrn2-5 double mutant (Fig. 2f). This double mutant was also more sensitive than prt6-1 to root waterlogging (Fig. 2g), a distinct but eco-physiologically relevant root hypoxia stress. Collectively, these data define an $\mathrm{O}_{2}$-sensitive Cys $2 \mathrm{~N}$-degron in VRN2. This $\mathrm{N}$-degron restricts VRN2 accumulation to meristems, which are proposed to be naturally hypoxic due to high metabolic activity and oxygen consumption ${ }^{30}$. VRN2 is stabilized throughout the plant in response to low- $\mathrm{O}_{2}$, where our genetic evidence suggests it contributes to hypoxic stress-survival. 
a

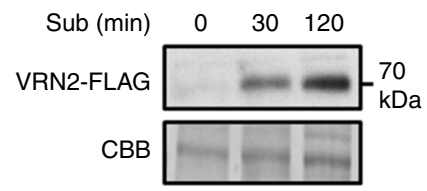

b

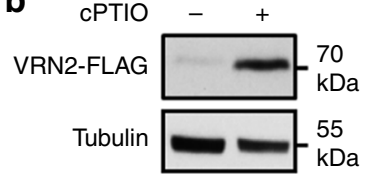

C

d

VRN2-GUS
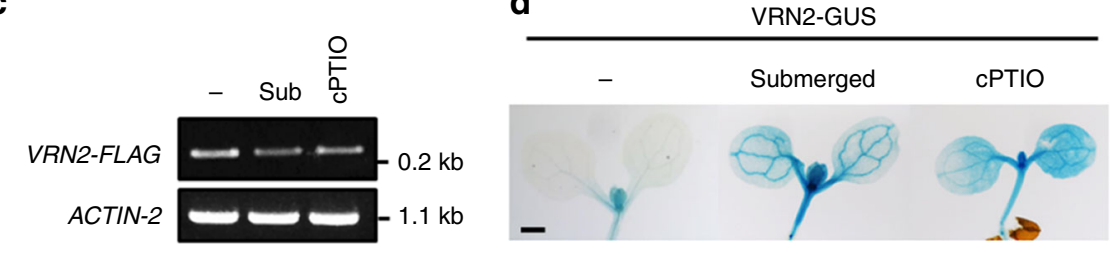

e
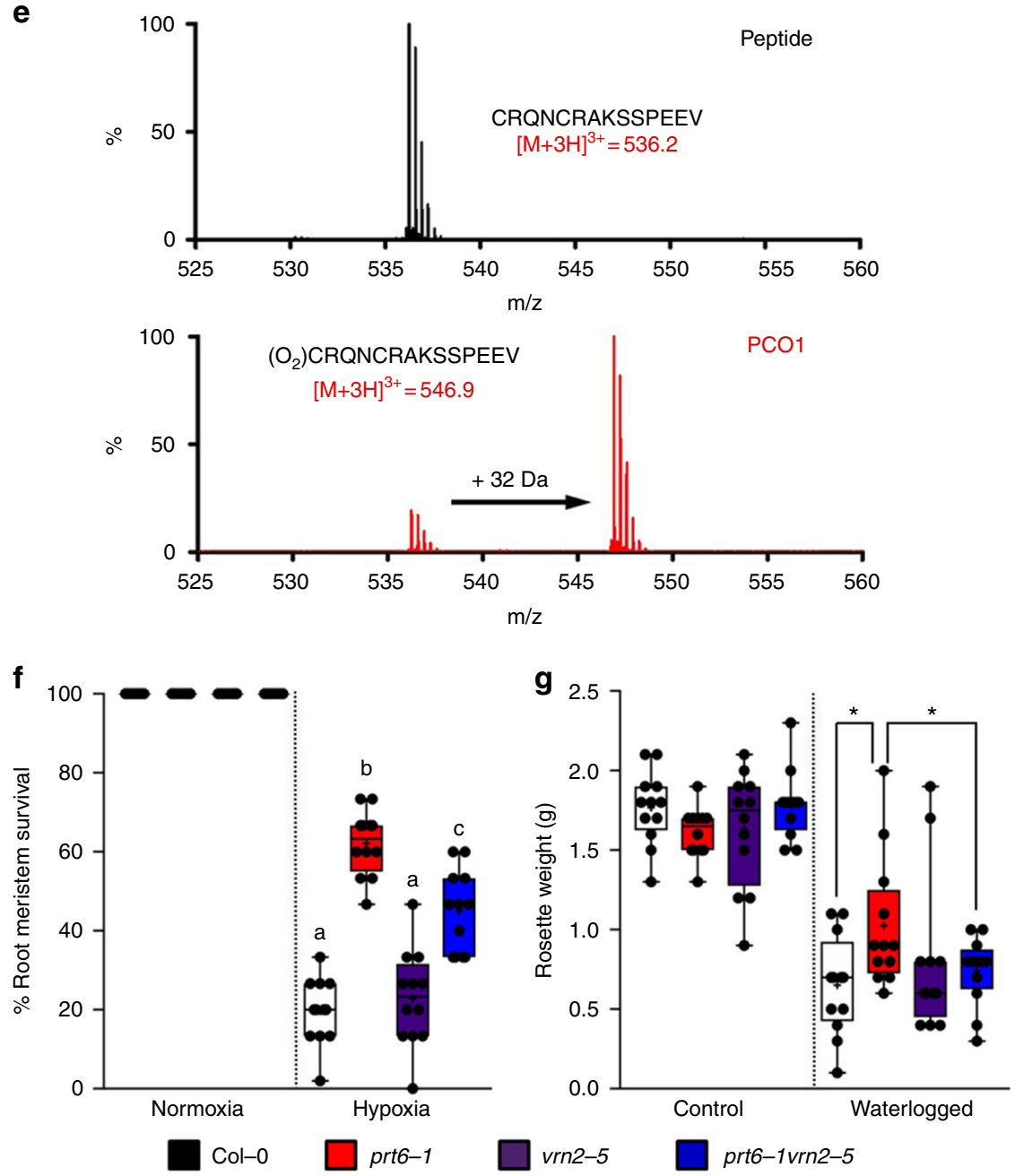

Fig. 2 VRN2 is regulated by $\mathrm{O}_{2}$ and $\mathrm{NO}$ availability and positively regulates hypoxia tolerance. a VRN2-FLAG protein accumulation during a submergence time course. $\mathbf{b}$ VRN2-FLAG protein accumulation \pm NO scavenger CPTIO. $\mathbf{c}$ mRNA levels of VRN2-FLAG \pm submergence or cPTIO treatment.

d Histochemical staining of 7-day-old Col-0 seedlings expressing WT VRN2-GUS \pm submergence-induced hypoxia or cPTIO treatment. Scale bar $500 \mu$ m. See also Supplementary Figure 2a for biological reps. e LC-MS spectra showing the VRN2 $2-15$ peptide species identified following incubation with or without recombinant PCO1. A product with a mass increase of $+32 \mathrm{Da}$, indicating $\mathrm{O}_{2}$-dependent Cys conversion to Cys-sulfinic acid, was only observed in the presence of PCO1. $\mathbf{f}$ Root tip survival data for 4-day-old Arabidopsis seedlings in normoxia or following $4 \mathrm{~h}$ hypoxia treatment $(n=15 \times 9$ for each genotype). Letters indicate one-way ANOVA; Tukey's test $(p<0.01)$. $\mathbf{g}$ Rosette weight of soil-grown plants \pm 21 days waterlogging treatment $(n=12$ for each genotype). ANOVA was not significant ( $p<0.08$ ). Pairwise $t$-tests (shown on graph; Col-0 vs prt6-1 and prt6-1 vs prt6-1 vrn2-5) were significant $\left({ }^{*} p<\right.$ 0.05). Box and whiskers plots show replicates (dots), max and min, 25th to 75 th percentiles, median and mean ( + ). Source data are provided as a Source Data file 
a

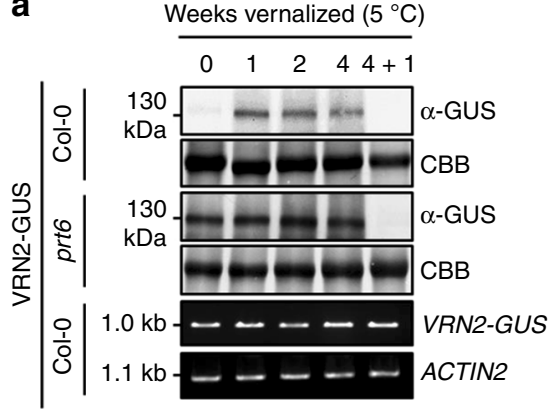

b

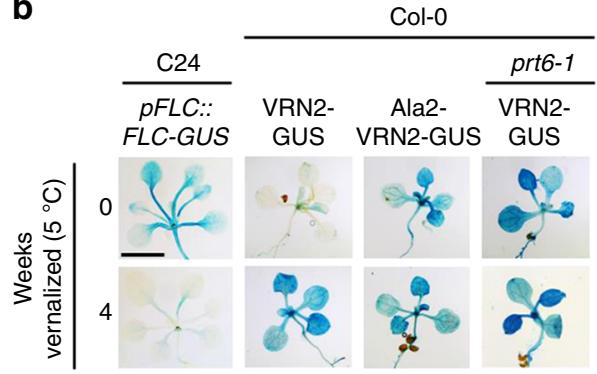

Fig. 3 The N-end rule pathway restricts VRN2 accumulation under non-vernalizing conditions. a Protein and mRNA levels of WT VRN2-GUS in vernalized (1-4 weeks) Col-0 and prt6-1 seedlings. $4+1$ refers to 1 week 'recovery' at $22^{\circ} \mathrm{C}$ following vernalization. b Histochemical staining of seedlings expressing pFLC::FLC-GUS (C24 background), and WT or mutant (Ala2) VRN2-GUS in Col-0 and prt6-1, \pm 4 weeks vernalization. Scale bar $5 \mathrm{~mm}$. See also Supplementary Figure $2 \mathrm{c}$ for biological reps. Source data are provided as a Source Data file

VRN2 abundance is restricted in the absence of cold. We next investigated whether the $\mathrm{N}$-end rule pathway regulates the known post-translational accumulation of VRN2 in response to longterm cold exposure, which is required for vernalization ${ }^{14,15}$. VRN2-GUS protein levels increased in Col-0 seedlings after 1 week at $5{ }^{\circ} \mathrm{C}$, without any detectable mRNA changes (Fig. 3a). In contrast, VRN2-GUS was already present at high levels in prt61 at $22^{\circ} \mathrm{C}$, showing only a small increase during cold treatment. This enhanced abundance was maintained throughout subsequent continued cold exposure. The spatial patterning of VRN2-GUS in seedlings after 4 weeks at $5{ }^{\circ} \mathrm{C}$ resembled that observed in prt6-1 (or mutant Ala2-VRN2-GUS in WT) at $22^{\circ} \mathrm{C}$, and was inversely correlated with cold-responsive pFLC::FLC$G_{U S}{ }^{31}$ repression observed in the vernalization-dependent C24 background (Fig. $3 \mathrm{~b}$ and Supplementary Figure 2c), in accordance with FLC being repressed by VRN2-PRC2. A return to $22^{\circ} \mathrm{C}$ after 4 weeks of cold exposure led to depletion of VRN2 in both WT and prt6-1, indicating the presence of other degron(s) in VRN2 independent of the Arg/N-end rule pathway. These data reveal that the N-end rule pathway limits VRN2 accumulation under non-vernalizing conditions.

Molecular responses to cold-exposure and hypoxia. The pattern of VRN2 protein accumulation in response to cold (Fig. 3b) is similar to that observed in response to submergence (Fig. 2d). In both conditions, the accumulation of VRN2 could be determined by reduced degradation via its $\mathrm{N}$-degron. We therefore considered possible mechanism(s) by which long-term cold exposure might promote stabilization of VRN2. Previous studies showed that ALCOHOL DEHYDROGENASE (ADH), a key hypoxia-induced protein, accumulates in cold-treated plants (including Arabidopsis, maize and rice $)^{32,33}$. Therefore, we investigated the potential link between gene-expression regulation in response to long-term cold and hypoxia. Using RNA seq analysis of non-vernalized vs 4-week-vernalized seedlings, we compared cold-induced transcripts with those induced by hypoxia ${ }^{21}$. This revealed a significant overlap in gene expression between the two conditions $(p<0.0015$; hypergeometric test $\left.{ }^{21}\right)$. Approximately $20 \%$ of hypoxia-upregulated genes $^{21}$ were upregulated by long-term cold treatment, including many of the core anaerobic-response genes that are universally induced by low- $\mathrm{O}_{2}{ }^{34}$ (Fig. 4a, b). Amongst the most highly upregulated transcripts were mRNAs critical for the survival of anaerobiosis (including $A D H 1$ and $P D C 1$ ), as well as VIN3, which interacts with VRN2-PRC2 to promote FLC silencing during vernalization $^{15,35}$, and which was previously also shown to be hypoxia-induced $^{36}$ (Fig. 4a, b). We investigated the relationship between vernalizing conditions and anaerobic gene expression in more detail, by analyzing the expression of $A D H 1$ using qPCR in different genetic backgrounds. $A D H 1$ was expressed at much higher levels in prt6-1 and prt6-1 vrn2-5 compared to WT and vrn2-5 under non-vernalizing conditions (Fig. 4c), corroborating previous studies showing ectopic accumulation of anaerobic genes in prt6-1 $1^{19,20}$. Furthermore, $A D H 1$ was still induced in vrn2-5 in response to cold treatment, and was hyper-induced in prt6-1 and prt6-1 vrn2-5 seedlings. This indicates that VRN2 accumulation in the cold does not significantly influence $A D H 1$ transcription, and that other factors, which are altered in prt6-1, promote anaerobic gene expression in response to cold.

Since ERFVII transcription factors are known substrates of the $\mathrm{N}$-end rule pathway that induce anaerobic gene expression in response to $\mathrm{O}_{2}$-deprivation ${ }^{19,20,26}$, we examined their role in regulating cold-triggered expression of hypoxia-associated genes. Similarly to VRN2, cold temperatures led to strong stabilization of the ERFVII proteins RAP2.3-HA and HRE2-HA (Fig. 4d). Furthermore, the cold-responsive hyper-induction of $A D H 1$ and $H B 1$ in prt6-1 was reverted in a prt6 erfVII ${ }^{27}$ sextuple mutant that lacks all 5 ERFVIIs (Fig. 4e, f and Supplementary Figure 6), suggesting that the cold-induced accumulation of ERFVIIs may control anaerobic gene induction. However, $A D H 1$ and $H B 1$ were still significantly upregulated by cold exposure in prt6 erfVII, albeit to a lesser extent than in WT (Fig. 4f and Supplementary Figure 6). These data suggest that upregulation of anaerobic genes in the cold could be potentiated by stabilized ERFVIIs, but that it is also controlled by a mechanism that is independent of ERFVIIs and the canonical hypoxia signalling pathway.

PRT6 expression is not significantly altered by cold temperature (Fig. 4b), indicating that enhanced stabilization of VRN2 and ERFVIIs is not a consequence of changes in N-recognin E3 ligase levels. However, we found in vitro PCO enzyme activities towards both VRN2 and ERFVII Nt-peptides were significantly reduced at $5{ }^{\circ} \mathrm{C}$ relative to $22^{\circ} \mathrm{C}$ (Fig. $4 \mathrm{~h}$ and Supplementary Figure $4 \mathrm{~b}-\mathrm{d})$. Furthermore, NO levels were significantly reduced in the roots of seedlings exposed to longterm cold conditions (Fig. 4h). Since NO-depletion can also promote accumulation of VRN2 (Fig. 2b, d) and ERFVIIs ${ }^{21}$, we propose that cold-exposure may induce conditions that enhance the stability of Cys-initiating $\mathrm{N}$-end rule substrates, including both VRN2 and the ERFVIIs, and that this stabilization may be partly responsible for the observed overlap in gene expression.

Angiosperm-specific recruitment of $\mathrm{Su}(\mathrm{z}) 12$ to the $\mathrm{N}$-end rule. We explored the conservation and evolutionary origins of the 
a

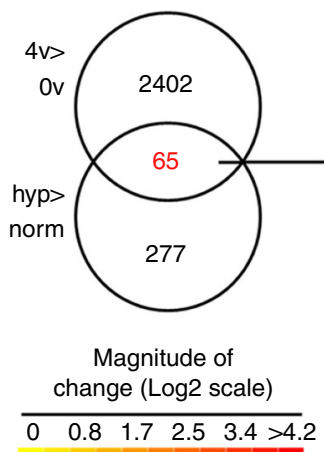

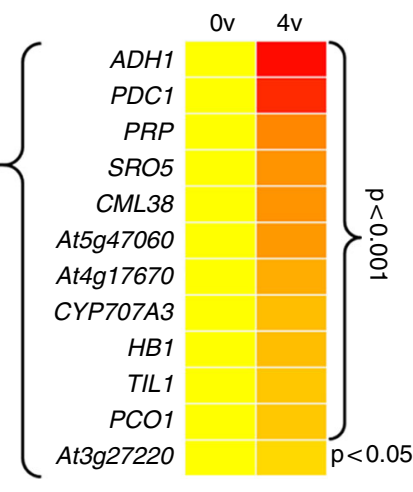

b

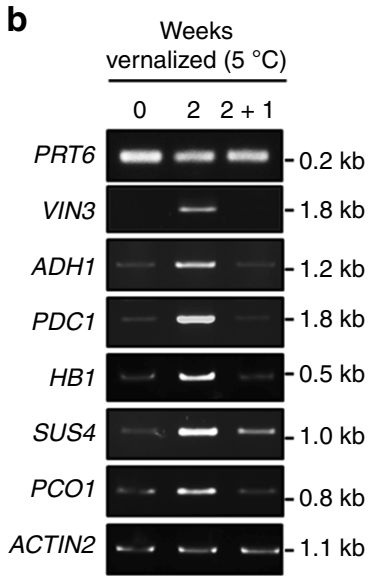

C
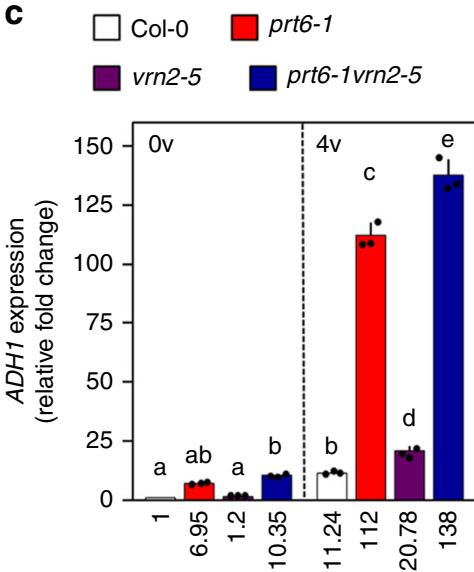

d

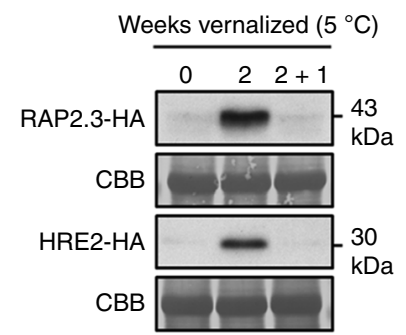

g

Fold reduction in $\mathrm{PCO}$ activities at $5^{\circ} \mathrm{C}$

\begin{tabular}{|c|c|c|}
\hline PCO & VRN2 & ERFVII \\
\hline 1 & 1.83 & 3.96 \\
\hline 2 & 2.26 & 7.85 \\
\hline 3 & 9.32 & 4.30 \\
\hline 4 & 2.39 & 3.36 \\
\hline 5 & 2.13 & 6.40 \\
\hline
\end{tabular}

e
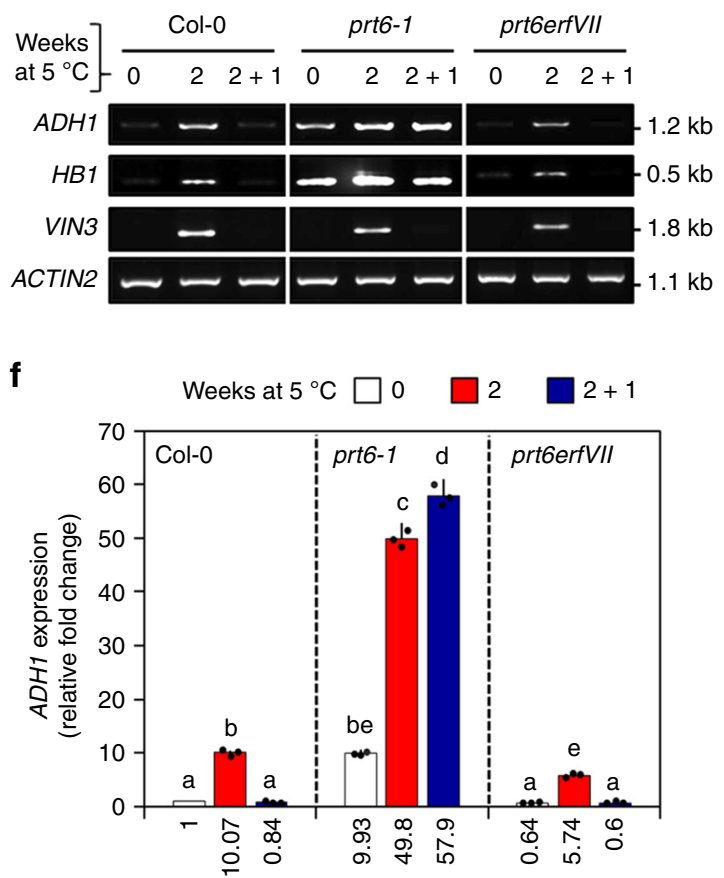

$\mathbf{h}_{0}$
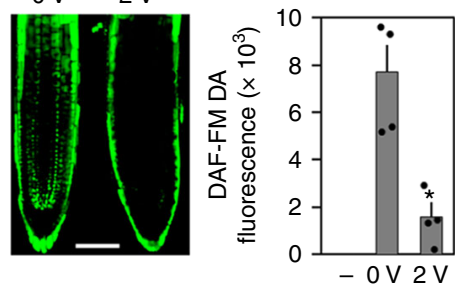

connection between PRC2 and the $\mathrm{N}$-end rule pathway. We identified and cloned an MC-initiating VRN2-like protein (HvEMF2c) from barley, a monocot species distantly related to Arabidopsis, and confirmed in vitro that it harbors a functional $\mathrm{N}$-degron (Fig. 5a). However, we did not identify MC-initiating $\mathrm{Su}(\mathrm{z}) 12$ homologs in basal land-plants or in animals. This suggests that $\mathrm{O}_{2}$-sensitive $\mathrm{Su}(\mathrm{z}) 12$ proteins are only found in angiosperms, although in some species, VRN2-like proteins might evade degradation despite carrying this $\mathrm{N}$-terminal sequence, similar to the SUB1A ERFVII in rice ${ }^{19}$.
We investigated how $\mathrm{Su}(\mathrm{z}) 12$ was recruited as a substrate of the $\mathrm{N}$-end rule pathway specifically in the flowering plant lineage. VRN2 is proposed to have evolved following duplication of an ancient EMF2-like $\mathrm{Su}(\mathrm{z}) 12$ gene $^{37,38}$. Alignment of Arabidopsis EMF2 and VRN2 protein sequences shows that the MC-N-terminus of VRN2 is equivalent to an internal MCdipeptide in the N-terminal cap region of EMF2 21 amino acid residues downstream of Met1 (Fig. 5b, c and Supplementary Figure 7), that is also present in EMF2-like proteins from gymnosperm taxa, but not in more basal land plants (Fig. 5c). 
Fig. 4 Transcriptional and post-translational responses to cold exposure. a Venn diagram showing overlap in upregulated genes between 4-weekvernalized vs non-vernalized $(4 v>0 v)$ and hypoxia vs normoxia ${ }^{21}$ (hyp > norm) treated seedlings ( $n=3$ per treatment). Heat map shows Log2 expression levels of 12 core anaerobic genes that are induced in both datasets (for numeric expression values, see Supplementary Data 1). b Semi-quantitative RT-PCR analysis of genes in Col-0 seedlings treated \pm 2 weeks vernalization, or 2 weeks vernalization followed by 1 week 'recovery' at $22^{\circ} \mathrm{C}(2+1)$. VIN3 induction serves as a control for cold exposure. c qPCR of ADH1 levels in Col-0, prt6-1, vrn2-5 and prt6-1 vrn2-5 seedlings with (4v) or without (0v) 4 weeks cold treatment. Each bar shows the mean of 3 biological reps (dots). Expression values are shown underneath and letters indicate one-way ANOVA; Tukey's test $(p<0.01)$. d Steady state protein levels of the ERFVII transcription factors RAP2.3-HA and HRE2-HA in seedlings vernalized as in (b). e Semiquantitative RT-PCR analysis of genes in Col-0, prt6-1 and prt6 erfVII seedlings treated as in (b). $\mathbf{f}$ qPCR of ADH1 levels in Col-0, prt6-1 and prt6 erfVII seedlings treated as in (b). Each bar shows the mean of 3 biological reps (dots). Expression values are shown underneath and letters indicate one-way ANOVA; Tukey's test $(p<0.01)$. g Fold reduction in the activities of PCO1-5 when incubated with $\mathrm{VRN}_{2-15}$ and ERFVII $2-15 \mathrm{~N}$-terminal peptides at $5{ }^{\circ} \mathrm{C}$ relative to $21^{\circ} \mathrm{C}$ (see also Supplementary Figure $4 \mathrm{~b}-\mathrm{d}$ ). $\mathbf{h}$ NO levels (measured as DAF-FM fluorescence) in non-vernalized (Ov) or 2-week-vernalized (2v) Col-0 roots. Scale bar $50 \mu \mathrm{m}$. Each bar shows the mean of 4 biological reps (dots). Error bars are SEM, ${ }^{\star} p<0.05$ ( $t$-test). Source data are provided as a Source Data file

This dipeptide sequence occurs in the basal angiosperm Amborella trichopoda and many other angiosperm taxa, but has diverged in some extant angiosperm groups, suggesting that it is not important for EMF2 function (Fig. $5 c$ and Supplementary Figure 8). Collectively these phylogenetic data suggest the internal MC dipeptide was fixed before angiosperms evolved, giving the capacity for an ancient EMF2-like protein to become an $\mathrm{O}_{2}$-regulated VRN2-like protein following removal of the Nt-cap. To test this, we cloned Arabidopsis EMF2 and showed full-length EMF2-HA was stable in vitro, but N-terminally truncated EMF2-HA (tEMF2-HA; Fig. 5b) was extremely unstable via its Cys2 residue (Fig. 5 d, e). This was confirmed in planta, where tEMF2-HA could only be detected in the prt6-1 mutant despite equivalent transgene mRNA levels in Col-0 (Fig. 5f). Moreover, this N-terminal truncation was sufficient to couple tEMF2-HA protein accumulation to cold exposure, similar to VRN2 (Fig. 5g). These data therefore support a mechanism for the evolutionary co-option of $\mathrm{Su}(\mathrm{z}) 12$ to the $\mathrm{N}$-end rule pathway in angiosperms, via gene duplication $^{37}$ and N-terminal truncation of an ancient EMF2-like protein.

\section{Discussion}

Here, we report that flowering plants have uniquely evolved a variant of the $\mathrm{Su}(\mathrm{z}) 12$ PRC2 component-VRN2-that is regulated by the $\mathrm{O}_{2}$ - and $\mathrm{NO}$-sensitive branch of the $\mathrm{Arg} / \mathrm{N}$-end rule pathway. This proteolytic regulation is required to prevent ectopic accumulation of VRN2 outside of meristems in the absence of external stimuli such as cold or hypoxia, despite constitutive VRN2 mRNA expression ${ }^{38}$. We show that both submergence and cold-exposure promote VRN2 accumulation. It will now be important to investigate in detail the connection between proteolytic control of VRN2 by the N-end rule pathway and the molecular changes that occur at known (e.g. FLC) and novel genome-wide targets of VRN2-PRC2.

Hypoxia frequently occurs in plants during development and in response to external abiotic stress such as flooding ${ }^{30,39}$. Reduced oxygen availability is transduced into transcriptional changes by the ERFVII transcription factors, which promote hypoxia tolerance through enhancing expression of key genes associated with anaerobiosis ${ }^{19,20}$. Here, we show that VRN2 stability is also regulated by $\mathrm{O}_{2}$, and that it could contribute to hypoxia stress-tolerance. As part of a chromatinmodifying complex, it is possible that VRN2 may be involved in the epigenetic control of gene expression linked to hypoxia acclimation, or NO signalling ${ }^{40}$. Together with ERFVIIs, this indicates that plants could modulate the transcriptional and epigenetic control of gene expression in response to $\mathrm{O}_{2}$ and NO availability by targeting functionally distinct proteins to the same proteolytic pathway, and suggests others remain to be identified ${ }^{41}$. Future studies examining the effect of physiological fluctuations of these gases on VRN2 accumulation, and the subsequent effect on the genome-wide targets of VRN2, may shed light on this. It is interesting to note that VIN3, another key protein that is transcriptionally induced by cold and essential for the vernalization response ${ }^{35}$, is also upregulated by hypoxia and may also therefore be required for VRN2 functions in response to low- $\mathrm{O}_{2}$.

VRN2 was originally identified as a positive regulator of vernalization, but a mechanism explaining its environmentallyinduced accumulation in response to cold temperatures has not been described ${ }^{14,15}$. We found that long-term cold inhibits VRN2 proteolysis and that the $\mathrm{N}$-end rule pathway is involved in restricting the accumulation of VRN2 in the absence of cold temperatures. Prolonged growth in the cold led not only to VRN2 accumulation, but also the induction of other molecular events that are also triggered by hypoxia, including stabilization of ERFVII transcription factors and upregulation of core anaerobic response genes. Exposure to cold temperatures has previously been shown to interfere with respiration through altering membrane fluidity properties in mitochondria, which initiates a shift to anaerobic metabolism ${ }^{32,33}$. Furthermore, transcriptional similarities between plants subjected to either hypoxic stress, or chemical inhibition of mitochondrial respiration, have also been reported ${ }^{42}$. Recently it was hypothesized that cold and hypoxia both represent an 'energy crisis' in plants, which may explain similar metabolic responses ${ }^{43}$.

Given the observed similarities in the cellular response to hypoxia and cold, we speculate that the accumulation of ERFVII transcription factors and VRN2 during cold-exposure could be related to biochemical changes that induce or mimic $\mathrm{O}_{2}$ limitation. We also investigated $\mathrm{O}_{2}$-independent mechanisms to explain cold-induced accumulation of VRN2 and ERFVIIs, and found that PCO activities and NO levels are both reduced at lower temperatures. Both of these situations could promote accumulation of $\mathrm{O}_{2}$-sensitive $\mathrm{N}$-end rule proteins even if $\mathrm{O}_{2}$ is present. Nevertheless, further work is needed to show if and how the N-end rule-dependent turnover of VRN2 and ERFVIIs is modified during cold exposure.

To conclude, our work has uncovered a proteolytic mechanism operating on the PRC2 machinery that functions specifically in flowering plants. This was achieved through the lineage-specific evolution of a conditional gas-sensitive N-degron in the $\mathrm{Su}(\mathrm{z}) 12$ component, perhaps representing a step toward PRC2 neofunctionalization in angiosperms. Given the key roles for VRN2 in regulating the coordination of flowering with seasonal cues, this coupling of $\mathrm{Su}(\mathrm{z}) 12$ to the $\mathrm{Arg} / \mathrm{N}$-end rule pathway may have facilitated a connection between environmental conditions and PRC2 activity, whilst also permitting further diversification of PRC2 function. 
a

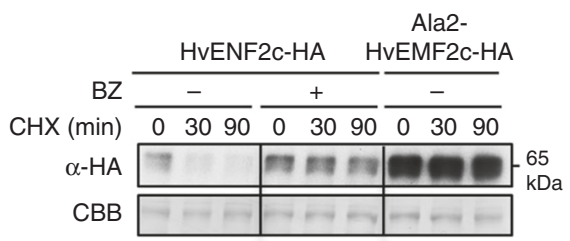

b

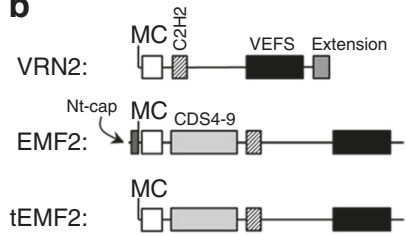

C

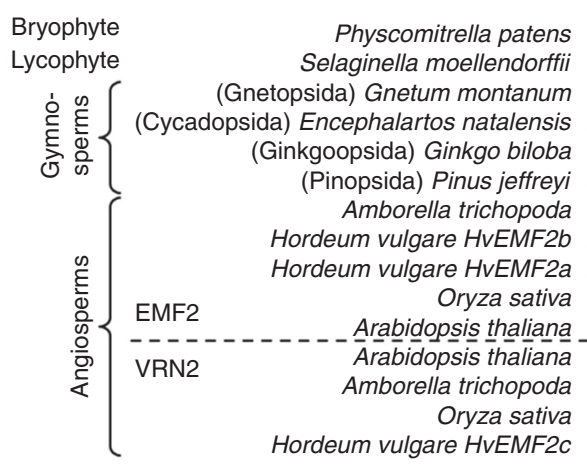

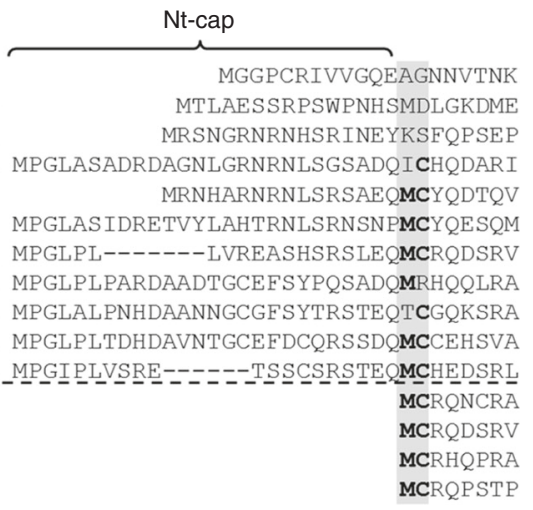

e d

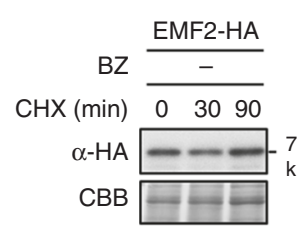

f

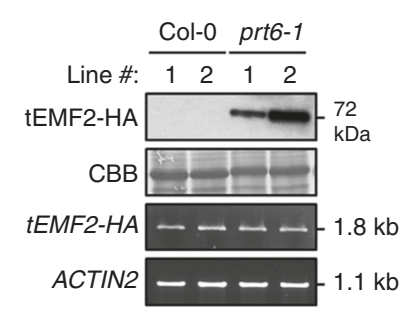

Ala-

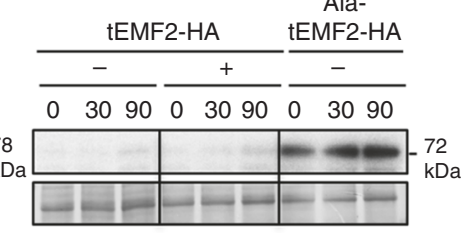

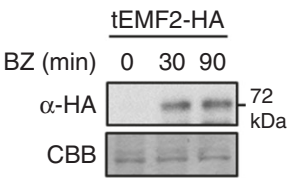

g Weeks vernalized $\left(5^{\circ} \mathrm{C}\right)$

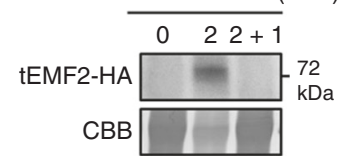

Fig. 5 Angiosperm-specific recruitment of Su(z)12 to the N-end rule pathway. a In vitro cycloheximide (CHX) chase of WT and mutant (Ala2) variants of the barley VRN2-like protein HvEMF2c ( \pm bortezomib; BZ). b Schematic showing the structure and key domains of Arabidopsis VRN2, EMF2 and truncated tEMF2. c Sequence alignment of the Nt of EMF2 and VRN2 proteins from land plants (above and below dashed line, respectively), showing the position of the MC dipeptide. For sequences see Supplementary Data 4. d In vitro CHX chase of full-length Arabidopsis EMF2-HA (initiating MP-), and WT or mutant (Ala2) tEMF2-HA, \pm BZ. e In vitro accumulation over time of tEMF2-HA expressed in rabbit reticulocyte when co-incubated with BZ, but without $\mathrm{CHX}$. $\mathbf{f}$ In vivo steady state protein and RNA levels of truncated WT tEMF2-HA in Col-0 vs prt6-1. Two independent transformants are shown. $\mathbf{g}$ Protein levels of tEMF2-HA in Col-0 during a vernalization timecourse. Source data are provided as a Source Data file

\section{Methods}

Plant growth and materials. A. thaliana (Arabidopsis) seedlings were obtained from the Arabidopsis Stock Centre (NASC), except for: VRN2-FLAG ${ }^{15}$ (from Dr. Chris Helliwell, CSIRO, Australia), and pFLC::FLC-GUS (C24 ecotype, containing $6 \mathrm{~kb}$ FLC-GUS construct ${ }^{31}$, from Dr. Candice Sheldon, CSIRO, Australia). The 35S::HRE2-HA and 35S::RAP2.3-HA transgenic lines, as well as prt6-1, ate1-2 ate21 and prt6-1 erfVII sextuple mutants were described previously ${ }^{19,21,27,28}$. Molecular characterisation of vrn2-5 (SALK_201153) is described in Supplementary Figure 5. Mutant combinations were generated by crossing, and confirmed by PCR genotyping and RT-PCR (primers in Supplementary Table 1). Seeds were surfaced sterilized in $20 \%$ Parazone, plated on half-strength Murashige and Skoog (MS) medium ( $1 \%$ agar, $\mathrm{pH} 5.7$, grown vertically), and stratified at $4{ }^{\circ} \mathrm{C}$ for a minimum of 2 days, before being transferred to long day (LD; $16 \mathrm{~h} \mathrm{~L}: 8 \mathrm{~h} \mathrm{D})$ condition under white fluorescent light $\left(90-100 \mu \mathrm{mol} \mathrm{m}^{-2} \mathrm{~s}^{-1}\right)$ at $22^{\circ} \mathrm{C}$, and transferred to soil after 2 weeks if necessary.

Plant phenotypic analyses. For the waterlogging assays, 12days old seedlings were transferred to soil and grown under neutral days (ND; $12 \mathrm{~h} \mathrm{~L}: 12 \mathrm{~h} \mathrm{D}$ ). Two replica trays were set up with genotypes distributed quasi-randomly. After 12 further days of growth in soil, one tray was subjected to waterlogging for 21 days (water level maintained at the soil surface), whilst the other (control) was watered as normal with good drainage. The fresh weight of each rosette was then measured. To measure root hypoxia survival, seedlings were grown under SD conditions at $20^{\circ} \mathrm{C}$ on vertical $1 / 2$ MS plates. After 4 days of growth, seedlings were placed into sealed desiccators. $100 \% \mathrm{~N}_{2}$ was flushed through the desiccators at a flow rate of $4 \mathrm{~L} / \mathrm{min}$, until oxygen levels fell to below $0.1 \%$. After hypoxia treatment, seedlings were returned to SD conditions for 3 further days. Roots were scored as dead if there was no growth following hypoxia treatment.

Construction of transgenic plants. To generate C-terminally GUS-tagged VRN2 driven by its native promoter, the full genomic DNA sequence (from approx. $2 \mathrm{~kb}$ upstream of the ATG and ending at the STOP codon) was amplified from seedling genomic DNA extracts using attB-flanked primers, and recombined into $\mathrm{pDO}$ NOR201 using gateway BP clonase (Invitrogen; 11789020). The C2A mutation was incorporated using $d p n I$-mediated site-directed mutagenesis, prior to mobilisation into the destination binary vector pGWB533 using LR clonase (Invitrogen; 11791100). To create 35S::tEMF2-HA (truncated EMF2) lines, tEMF2 was amplified from seedling cDNA, directionally cloned into $\mathrm{pE} 2 \mathrm{c}$ and mobilised into pB2GW7. For all cloning primers see Table S1. Transformation into Agrobacterium tumefaciens (strain GV3101 pMP90) and Arabidopsis was performed according to established protocols. At least 8 independent transgenic plants were selected for each construct; data from $2-3$ independent $\mathrm{T}_{3}$ homozygous lines are shown. 
In vivo protein stability analyses. Total protein was extracted from un-treated or treated 7-day-old (or appropriately vernalized) seedlings by grinding up frozen samples directly into SDS protein extraction buffer $(125 \mathrm{mM}$ Tris-HCl pH 8.8, $1 \%$ $(\mathrm{w} / \mathrm{v}) \mathrm{SDS}, 10 \%(\mathrm{v} / \mathrm{v})$ glycerol, $\left.50 \mathrm{mM} \mathrm{Na}_{2} \mathrm{~S}_{2} \mathrm{O}_{5}\right)$ using a micropestle, before centrifugating and determining protein concentration in the supernatant using the BioRad DC Protein Assay (Bio-Rad) ${ }^{19}$. To assess the effects of submergenceinduced hypoxia on in vivo protein stability, seedlings were immersed in $2 \mathrm{ml}$ Eppendorf tubes filled with degassed water, sealed with parafilm, and incubated in the dark at room temperature for the times indicated. To test the effects of nitric oxide scavenging, seedlings were incubated in liquid $1 / 2$ MS with $200 \mu \mathrm{M}$ cPTIO for $6 \mathrm{~h}$ (Enzo life science; ALX-430-001) ${ }^{19,21}$. For cycloheximide-chase assays, seedlings were transferred to liquid $1 / 2 \mathrm{MS}$ in 6-well microtiter plates supplemented with $100 \mathrm{mM}$ cycloheximide (Sigma-Aldrich; C4859), $100 \mathrm{mM}$ bortezomib (ApexBio technology; A2614), both, or appropriate solvent controls. Seedlings were then incubated at $22{ }^{\circ} \mathrm{C}$ in the light with gentle shaking, and harvested at stated time points for protein/RNA extraction (in liquid nitrogen) or GUS histochemical staining.

Histochemical staining. For histochemical analysis of $\beta$-Glucuronidase (GUS) enzyme activity, transgenic Arabidopsis tissues were incubated in a buffer containing: phosphate buffer $(100 \mathrm{mM}) \mathrm{pH} 7.0$, potassium ferricyanide $(2 \mathrm{mM})$, potassium ferrocyanide $(2 \mathrm{mM})$, Triton X-100 (0.1\% v/v) and X-Gluc solution (5-bromo-4-chloro3 -indolyl-beta-D-glucuronic acid, cyclohexylammonium salt, X-GLUC Direct) ( $1 \mathrm{mM}$ ). Samples were then incubated at $37^{\circ} \mathrm{C}$ in the buffer for $4-8 \mathrm{~h}$. Seedlings were cleared and fixed in 3:1 ethanol:acetic acid before mounting in Hoyer's solution ( $30 \mathrm{~g}$ gum Arabic, $200 \mathrm{~g}$ chloral hydrate, $20 \mathrm{~g}$ glycerol, $50 \mathrm{ml}$ water) before imaging on a light microscope.

In vitro stability assays. To generate protein-HA fusions driven by the T7 promoter, cDNAs were PCR amplified from Arabidopsis or barley total CDNA and directionally cloned into a modified version of the pTNT (Invitrogen) expression vector $\left(\mathrm{pTNT}^{\mathrm{x}} \mathrm{xHA}^{19}\right)$. N-terminal variations were incorporated by changing the forward primer (primers in Supplementary Table 1). Cycloheximide-chase assays were then performed using the TNT T7 Coupled Reticulocyte Lysate system (Promega; L4610) $^{19,44}$, using $50 \mu \mathrm{M}$ bortezomib in place of MG132. For Fig. 5e, $50 \mu \mathrm{M}$ bortezomib was added at the beginning of the reaction and no cycloheximide was used. All assays were performed at least three times.

Immunoblotting and relative quantification. Equal total protein amounts were resolved by SDS-PAGE, and were transferred to PVDF using a MiniTrans-Blot electrophoretic transfer cell (Bio-Rad). Membranes were probed with primary antibodies at the following dilutions: anti-HA (Sigma-Aldrich; H3663), 1:2000 anti-GUS (Sigma-Aldrich; G5420), 1:1000; anti-FLAG (Sigma-Aldrich; F1804), 1:1000. HRP-conjugated anti-mouse or rabbit secondary antibodies (Santa Cruz; sc-358914 and sc-2004) were used at a titre of 1:10,000. Immunoblots were developed to film using ECL western blotting substrate (Pierce). For quantification of steady state levels in different mutant backgrounds, relative pixel density of protein bands from three independent lines for each transgene was assessed using imageJ. All blots were performed at least three times.

Analysing the activities of AtPCO1-5 in vitro. A. thaliana PCOs 1 to 5 were expressed and purified by sequential steps of immobilized nickel affinity and size exclusion chromatography as previously described ${ }^{45}$. The activities of each AtPCO were examined by incubating $200 \mu \mathrm{M}$ of peptide corresponding to the first 14 amino acids of the methionine excised N-terminus of VRN2 (VRN2 $2_{2-15}$ ) or RAP2.2/2.12 (RAP2 $2-15$ ) with and without $0.1-0.8 \mu \mathrm{M}$ enzyme in a bench top thermocycler (Eppendorf) at 5 or $22^{\circ} \mathrm{C}$ under aerobic conditions. Time points were taken at regular intervals by quenching the reaction 1 in 10 with $1 \%$ formic acid, allowing oxidation to be monitored by ultra-high performance chromatography (UPLC)-mass spectrometry (MS). Turnover was quantified by comparing the areas underneath the product and substrate ions extracted from the total ion current chromatogram. UPLC-MS measurements were obtained using an Acquity UPLC system coupled to a Xevo G2-S Q-ToF mass spectrometer (Waters) operated in positive electrospray mode. Instrument parameters, data acquisition and data processing were controlled by Masslynx 4.1 with source conditions adjusted to maximise sensitivity and minimise fragmentation. Samples were injected on to a Chromolith Performance RP-18e 100-2 mm column (Merck) heated to $40^{\circ} \mathrm{C}$ and eluted at $0.3 \mathrm{ml} / \mathrm{min}$ using a gradient of $95 \%$ deionized water supplement with $0.1 \%$ $(\mathrm{v} / \mathrm{v})$ formic acid to $95 \%$ acetonitrile.

NO detection by fluorescence microscopy. Intracellular NO levels were visualized using DAF-FM diacetate ( $7^{\prime}$-difluorofluorescein diacetate, Bio-Connect). Seedlings were incubated in the dark for $15 \mathrm{~min}$ in $10 \mathrm{mM}$ Tris- $\mathrm{HCl}$ buffer $(\mathrm{pH}$ 7.4) containing $50 \mu \mathrm{M}$ DAF-FM DA and subsequently washed twice for $5 \mathrm{~min} 10$ $\mathrm{mM}$ Tris- $\mathrm{HCl}$ buffer ( $\mathrm{pH}$ 7.4). Several roots of all treatments were mounted on the same slide to allow direct comparison. Fluorescence was visualized using a Zeiss Observer Z1 LSM7 confocal microscope with excitation at $488 \mathrm{~nm}$ and emission $520 \mathrm{~nm}$. Roots (0v) incubated and mounted in $10 \mathrm{mM}$ Tris- $\mathrm{HCl}$ buffer (pH 7.4) without DAF-FM DA were used as a negative control to set background values.
Within experiments, pinhole, gain, laser power and detector offset were identical for all slides. Mean DAF-FM DA fluorescence pixel intensity within the root was determined using ICY software (http://icy.bioimageanalysis.org/).

Phylogenetic analyses. EMF2 and VRN2-like sequences from flowering plants (NCBI organism ID: angiosperms [taxid:3398]) were obtained using the Arabidopsis EMF2 or VRN2 protein sequence, and sequence similarity assessed using the NCBI (https://blast.ncbi.nlm.nih.gov) Protein Basic Local Alignment Search Tool (BLASTP). Protein sequences representing diverse angiosperm clades (Supplementary Data 2 and Supplementary Data 3) were aligned using MUltiple Sequence Comparison by Log-Expectation (MUSCLE) ${ }^{46}$ in the programme MacVector Inc. (NC, USA). Weblogo 3.0 was used to obtain a graphical representation of amino acid proportions at the amino-terminus of VRN $2^{47}$. EMF2-like sequences from non-angiosperm plants were obtained either from genome sequences or from sequenced transcriptomes of other species via the $1 \mathrm{kp}$ project ${ }^{48-58}$ (Supplementary Data 4). Multiple sequence alignments were carried out as described above.

Reverse transcriptase PCR and qPCR. For semi-quantitative RT-PCR, RNA was extracted from seedlings using an RNEasy plant mini kit (Qiagen; 74904) and converted to cDNA with Superscript II Reverse transcriptase (Invitrogen; 18064014) using OligodT primers. PCRs were then performed with gene-specific or transgene-specific (i.e. gene-specific forward, tag-specific reverse) primers, and ACTIN-2 was amplified for use as a loading control. For quantitative assessment of FLC expression, RNA was extracted from 11-day-old seedlings grown at $22^{\circ} \mathrm{C}$ and converted to CDNA as described above. Real-time quantitative RT-PCR was performed in triplicate using Brilliant III UF MM SYBR QPCR Low ROX master mix (Agilent; 600892) on an AriaMx Real-Time PCR system (Agilent) according to manufacturer's instructions. Relative transcript levels for $A D H 1$ or $H B 1$ were determined by normalization to ACTIN. The control value was converted to 1 and relative fold change for other lines and treatments calculated. Data shown are mean of three biological repeats. Error bars indicate standard deviation. For primer sequences see Supplementary Table 1.

RNA sequencing. RNA was extracted from non-vernalized (0v) or 4 weeks vernalized (4v) seedlings grown on vertical $1 / 2$ MS plates as described above. Three biological replicates for each treatment were used for subsequent RNA sequencing, which was carried out at Glasgow Polyomics (www.polyomics.gla.ac.uk). An Initial QC was carried out using a nanodrop (to measure concentration) and the quality was tested on the Agilent Bioanalyser on a RNA Nano chip to ensure RIN values were above 8 . The library was then prepared using the Illumina TruSeq mRNA kit (polyA selection), before being analysed using the Qubit and the bioanalyser HS DNA chip. Samples were pooled and sequenced on the HiSeq 4000. 150 bp reads $(75 \times 2$ per fragment) were generated in fastq format, and aligned to the Arabidopsis genome (TAIR10), and then analysed using Kallisto ${ }^{59}$ (https://pachterlab. github.io/kallisto/about) before being processed using the R package, DESeq2 ${ }^{60}$ (https://bioconductor.org/packages/release/bioc/html/DESeq2.html). DESeq2 was used to compare pairs of samples and generate the final set of excel spreadsheets showing fold change differences. For subsequent analyses the gene list was filtered for genes upregulated in $4 \mathrm{v}$ by at least 1.5 fold, with a $p$ value $<0.05$. Venn diagrams of overlapping gene sets were generated using Venny 2.1, and expression heatmaps created using the BAR HeatMapper tool (https://bar.utoronto.ca/ntools/ cgi-bin/ntools_heatmapper.cgi). The hypergeometric test used to determine the significance of overlap measures the probability of observing a 65 or more gene overlap between two lists of length 2101 and 342 chosen at random out of 15,898 genes. 2101 is the number of cold upregulated genes that are also on the ATH1 microarray used in Gibbs et al. ${ }^{19}$, and 15,898 is the genes that were induced by hypoxia in Gibbs et al. ${ }^{19}$.

Reporting summary. Further information on experimental design is available in the Nature Research Reporting Summary linked to this article.

\section{Data availability}

RNA-seq data is available at NCBI GEO database with accession code GSE123459. The source data underlying all figures can be found in the Source Data file.

Received: 11 June 2018 Accepted: 4 December 2018

Published online: 21 December 2018

\section{References}

1. Simon, J. A. \& Kingston, R. E. Mechanisms of polycomb gene silencing: knowns and unknowns. Nat. Rev. Mol. Cell Biol. 10, 697-708 (2009).

2. Schwartz, Y. B. \& Pirrotta, V. A new world of Polycombs: unexpected partnerships and emerging functions. Nat. Rev. Genet. 14, 853-864 (2013).

3. Derkacheva, M. \& Hennig, L. Variations on a theme: Polycomb group proteins in plants. J. Exp. Bot. 65, 2769-2784 (2014). 
4. Margueron, R. \& Reinberg, D. The Polycomb complex PRC2 and its mark in life. Nature 469, 343-349 (2011).

5. Mozgova, I., Kohler, C. \& Hennig, L. Keeping the gate closed: functions of the polycomb repressive complex PRC2 in development. Plant J. 83, 121-132 (2015).

6. Yuan, W. et al. Dense chromatin activates Polycomb repressive complex 2 to regulate H3 lysine 27 methylation. Science 337, 971-975 (2012).

7. Forderer, A., Zhou, Y. \& Turck, F. The age of multiplexity: recruitment and interactions of Polycomb complexes in plants. Curr. Opin. Plant Biol. 29, 169-178 (2016).

8. Hennig, L. \& Derkacheva, M. Diversity of Polycomb group complexes in plants: same rules, different players? Trends Genet. 25, 414-423 (2009).

9. Yadegari, R. et al. Mutations in the FIE and MEA genes that encode interacting polycomb proteins cause parent-of-origin effects on seed development by distinct mechanisms. Plant Cell 12, 2367-2382 (2000).

10. Yoshida, N. et al. EMBRYONIC FLOWER2, a novel polycomb group protein homolog, mediates shoot development and flowering in Arabidopsis. Plant Cell 13, 2471-2481 (2001).

11. Roszak, P. \& Kohler, C. Polycomb group proteins are required to couple seed coat initiation to fertilization. Proc. Natl Acad. Sci. USA 108, 20826-20831 (2011).

12. Ikeuchi, M. et al. PRC2 represses dedifferentiation of mature somatic cells in Arabidopsis. Nat. Plants 1, 15089 (2015).

13. de Lucas, M. et al. Transcriptional regulation of Arabidopsis Polycomb Repressive Complex 2 coordinates cell-type proliferation and differentiation. Plant Cell 28, 2616-2631 (2016).

14. Gendall, A. R., Levy, Y. Y., Wilson, A. \& Dean, C. The VERNALIZATION 2 gene mediates the epigenetic regulation of vernalization in Arabidopsis. Cell 107, 525-535 (2001).

15. Wood, C.C. et al. The Arabidopsis thaliana vernalization response requires a polycomb-like protein complex that also includes VERNALIZATION INSENSITIVE 3. Proc. Natl Acad. Sci. USA 103, 14631-14636 (2006)

16. Varshavsky, A. The $\mathrm{N}$-end rule pathway and regulation by proteolysis. Protein Sci. 20, 1298-1345 (2011).

17. Gibbs, D. J., Bacardit, J., Bachmair, A. \& Holdsworth, M. J. The eukaryotic Nend rule pathway: conserved mechanisms and diverse functions. Trends Cell Biol. 24, 603-611 (2014).

18. Bachmair, A., Finley, D. \& Varshavsky, A. In vivo half-life of a protein is a function of its amino-terminal residue. Science 234, 179-186 (1986).

19. Gibbs, D. J. et al. Homeostatic response to hypoxia is regulated by the $\mathrm{N}$-end rule pathway in plants. Nature 479, 415-418 (2011).

20. Licausi, F. et al. Oxygen sensing in plants is mediated by an $\mathrm{N}$-end rule pathway for protein destabilization. Nature 479, 419-422 (2011).

21. Gibbs, D. J. et al. Nitric oxide sensing in plants is mediated by proteolytic control of group VII ERF transcription factors. Mol. Cell 53, 369-379 (2014).

22. $\mathrm{Hu}, \mathrm{R}$. G. et al. The $\mathrm{N}$-end rule pathway as a nitric oxide sensor controlling the levels of multiple regulators. Nature 437, 981-986 (2005).

23. Kwon, Y. T. et al. An essential role of N-terminal arginylation in cardiovascular development. Science 297, 96-99 (2002).

24. Weits, D. A. et al. Plant cysteine oxidases control the oxygen-dependent branch of the N-end-rule pathway. Nat. Commun. 5, 3425 (2014).

25. White, M. D. et al. Plant cysteine oxidases are dioxygenases that directly enable arginyl transferase-catalysed arginylation of $\mathrm{N}$-end rule targets. Nat. Commun. 8, 14690 (2017)

26. Gibbs, D. et al. Group VII ethylene response factors co-ordinate oxygen and nitric oxide signal transduction and stress responses in plants. Plant Physiol. 169, 23-31 (2015).

27. Abbas, M. et al. Oxygen sensing coordinates photomorphogenesis to facilitate seedling survival. Curr. Biol. 25, 1483-1488 (2015).

28. Vicente, J. et al. The Cys-Arg/N-end rule pathway is a general sensor of abiotic stress in flowering plants. Current Biology 27, 3183-3190 (2017).

29. Vicente, J. et al. Distinct branches of the N-end rule pathway modulate the plant immune response. The New Phytologist. Preprint at: https://www.ncbi. nlm.nih.gov/pubmed/30117535, https://doi.org/10.1111/nph.15387 (2018).

30. Considine, M. J. et al. Learning to breathe: developmental phase transitions in oxygen status. Trends Plant Sci. 22, 140-153 (2017).

31. Sheldon, C. C. et al. Resetting of FLOWERING LOCUS C expression after epigenetic repression by vernalization. Proc. Natl Acad. Sci. USA 105, 2214-2219 (2008).

32. Jarillo, J. A., Leyva, A., Salinas, J. \& Martinez-Zapater, J. M. Low temperature induces the accumulation of alcohol dehydrogenase mRNA in Arabidopsis thaliana, a chilling-tolerant plant. Plant Physiol. 101, 833-837 (1993).

33. Christie, P. J., Hahn, M. \& Walbot, V. Low-temperature accumulation of alcohol dehydrogenase-1 mRNA and protein activity in maize and rice seedlings. Plant Physiol. 95, 699-706 (1991).
34. Mustroph, A. et al. Profiling translatomes of discrete cell populations resolves altered cellular priorities during hypoxia in Arabidopsis. Proc. Natl Acad. Sci. USA 106, 18843-18848 (2009).

35. Sung, S. \& Amasino, R. M. Vernalization in Arabidopsis thaliana is mediated by the PHD finger protein VIN3. Nature 427, 159-164 (2004).

36. Bond, D. M., Wilson, I. W., Dennis, E. S., Pogson, B. J. \& Jean Finnegan, E. VERNALIZATION INSENSITIVE 3 (VIN3) is required for the response of Arabidopsis thaliana seedlings exposed to low oxygen conditions. Plant J. 59, 576-587 (2009).

37. Chen, L. J., Diao, Z. Y., Specht, C. \& Sung, Z. R. Molecular evolution of VEFdomain-containing PcG genes in plants. Mol. Plant 2, 738-754 (2009).

38. Qiu, Y., Liu, S. L. \& Adams, K. L. Concerted divergence after gene duplication in Polycomb repressive complexes. Plant Physiol. 174, 1192-1204 (2017).

39. Bailey-Serres, J. et al. Making sense of low oxygen sensing. Trends Plant Sci. 17, 129-138 (2012).

40. Domingos, P., Prado, A. M., Wong, A., Gehring, C. \& Feijo, J. A. Nitric oxide: a multitasked signaling gas in plants. Mol. Plant 8, 506-520 (2014).

41. Holdsworth, M. J. First hints of new sensors. Nat. Plants 3, 767-768 (2017).

42. Wagner, S., Van Aken, O., Elsasser, M. \& Schwarzlander, M. Mitochondrial energy signaling and its role in the low-oxygen stress response of plants. Plant Physiol. 176, 1156-1170 (2018).

43. Greenway, H. \& Armstrong, W. Energy-crises in well-aerated and anoxic tissue: does tolerance require the same specific proteins and energy-efficient transport? Funct. Plant Biol. 45, 877-894 (2018).

44. Mendiondo, G. M. et al. Enhanced waterlogging tolerance in barley by manipulation of expression of the $\mathrm{N}$-end rule pathway E3 ligase PROTEOLYSIS6. Plant Biotechnol. J. 14, 40-50 (2015).

45. White, M. D., Kamps, J., East, S., Taylor Kearney, L. J. \& Flashman, E. The plant cysteine oxidases from Arabidopsis thaliana are kinetically tailored to act as oxygen sensors. J. Biol. Chem. https://doi.org/10.1074/jbc.RA118.003496 (2018).

46. Edgar, R. C. MUSCLE: multiple sequence alignment with high accuracy and high throughput. Nucleic Acids Res. 32, 1792-1797 (2004).

47. Crooks, G. E., Hon, G., Chandonia, J. M. \& Brenner, S. E. WebLogo: a sequence logo generator. Genome Res. 14, 1188-1190 (2004).

48. Albert, V. A. et al. The Amborella genome and the evolution of flowering plants. Science 342, 1467 (2013).

49. Banks, J. A. et al. The Selaginella genome identifies genetic changes associated with the evolution of vascular plants. Science 332, 960-963 (2011).

50. Bowman, J. L. et al. Insights into land plant evolution garnered from the Marchantia polymorpha genome. Cell 171, 287 (2017).

51. Goff, S. A. et al. A draft sequence of the rice genome (Oryza sativa L. ssp japonica). Science 296, 92-100 (2002).

52. Guan, R. et al. Draft genome of the living fossil Ginkgo biloba. Gigascience 5, 49 (2016).

53. Kaul, S. et al. Analysis of the genome sequence of the flowering plant Arabidopsis thaliana. Nature 408, 796-815 (2000).

54. Mascher, M. et al. A chromosome conformation capture ordered sequence of the barley genome. Nature 544, 426 (2017).

55. Rensing, S. A. et al. The Physcomitrella genome reveals evolutionary insights into the conquest of land by plants. Science 319, 64-69 (2008).

56. Roodt, D. et al. Evidence for an ancient whole genome duplication in the cycad lineage. PloS ONE 12, e0184454 (2017).

57. Wan, T. et al. A genome for gnetophytes and early evolution of seed plants. Nat. Plants 4, 82-89 (2018).

58. Matasci, N. et al. Data access for the 1,000 plants $(1 \mathrm{KP})$ project. Gigascience 3 , 17 (2014)

59. Bray, N. L., Pimentel, H., Melsted, P. \& Pachter, L. Near-optimal probabilistic RNA-seq quantification. Nat. Biotechnol. 34, 525-527 (2016).

60. Love, M. I., Huber, W. \& Anders, S. Moderated estimation of fold change and dispersion for RNA-seq data with DESeq2. Genome Biol. 15, 550 (2014).

\section{Acknowledgements}

We thank C. Neal Stewart (University of Tennessee) for contributing Pinus jeffreyi data to the $1 \mathrm{KP}$ project, Julietta Marquez for technical assistance, Simon Pearce for help with statistics, and Glasgow Polyomics (polyomics.gla.ac.uk) for their assistance with the RNAseq investigation. This work was supported by the Biotechnology and Biological Sciences Research Council [Grant numbers BB/M020568/1, BB/M007820/1, BB/ M002268/1, BB/M024458/1], and a European Research Council grant (ERC Starting Grant 715441-GasPlaNt) to D.J.G. The NWO-ALW programme Graduate school Horticulture and Starting materials (EPS2 3c 195) supported the work of S.H.

\section{Author contributions}

D.J.G. and M.J.H. conceived the project and designed the research. D.J.G., M.J.H., H.M.T., A.-M.L., M.B., M.D.W., S.H., C.S., S.L.M., R.O., C.D. and T.B. performed research. D.J.G., 
M.J.H., M.D.W., Z.P., L.A.C.J.V. and E.F. analysed data. D.J.G. and M.J.H. wrote the manuscript.

\section{Additional information}

Supplementary Information accompanies this paper at https://doi.org/10.1038/s41467018-07875-7.

Competing interests: The authors declare no competing interests.

Reprints and permission information is available online at http://npg.nature.com/ reprintsandpermissions/

Journal peer review information: Nature Communications thanks the anonymous reviewers for their contribution to the peer review of this work.
Publisher's note: Springer Nature remains neutral with regard to jurisdictional claims in published maps and institutional affiliations.

(c) (i) Open Access This article is licensed under a Creative Commons Attribution 4.0 International License, which permits use, sharing, adaptation, distribution and reproduction in any medium or format, as long as you give appropriate credit to the original author(s) and the source, provide a link to the Creative Commons license, and indicate if changes were made. The images or other third party material in this article are included in the article's Creative Commons license, unless indicated otherwise in a credit line to the material. If material is not included in the article's Creative Commons license and your intended use is not permitted by statutory regulation or exceeds the permitted use, you will need to obtain permission directly from the copyright holder. To view a copy of this license, visit http://creativecommons.org/ licenses/by/4.0/.

(C) The Author(s) 2018 